Res., Soc. Dev. 2019; 8(10):e048101325

ISSN 2525-3409 | DOI: http://dx.doi.org/10.33448/rsd-v8i10.1325

\title{
Percepciones sobre la educación ambiental en el Instituto Superior de Educación
}

Percepções sobre a educação ambiental no Instituto Superior de Educação

Perceptions on environmental education at the Higher Education Institute

Recebido: 20/06/2019 | Revisado: 21/06/2019 | Aceito: 26/06/2019 | Publicado: 27/06/2019

Leonardo Mendes Bezerra

ORCID: https://orcid.org/ 0000-0002-9781-0047

Universidade Estadual do Maranhão, Campus Balsas, Brasil.

E-mail: lydimo@live.com

\section{Resumen}

El artículo presentado, fruto de una investigación, tuvo como objetivo identificar y describir las acciones y los modos de ver la educación ambiental (EA) en los cursos de licenciatura del Instituto Superior de Educación (ISE), institución vinculada al Centro de Universitario de Anápolis (UniEvangélica). Por tratarse de un estudio de caso, se utilizó el cuestionario como instrumento para recoger datos con los docentes y discentes de los cursos de licenciatura en química, matemáticas, biología, letras, historia y pedagogía de dicha institución. El resultado fue descriptivo y exploratorio en que se discutió cualitativamente bajo la luz de la literatura sobre medio ambiente y EA. Como resultado del estudio, en la visión de los sujetos involucrados que el ISE ha adquirido una mayor percepción de las cuestiones ambientales, a través de las actividades e investigaciones que enfocan las temáticas del medio ambiente y la educación ambiental.

Palabras clave: Sociedad. Medio ambiente. Educación.

\section{Resumo}

$\mathrm{O}$ artigo, ora apresentado, fruto de uma pesquisa, teve como escopo identificar e descrever as ações e os modos de ver a educação ambiental (EA) nos cursos de licenciatura do Instituto Superior de Educação (ISE), instância vinculada ao Centro de Universitário de Anápolis (UniEvangélica). Por se tratar de um estudo de caso, utilizou-se o questionário como instrumento para coletar dados com os docentes e discentes dos cursos de licenciatura em química, matemática, biologia, letras, história e pedagogia da referida instituição. O resultado foi descritivo e exploratório em que se discutiu-se qualitativamente sob a luz da literatura sobre meio ambiente e EA. Como resultado do estudo, na visão dos sujeitos envolvidos que 
Res., Soc. Dev. 2019; 8(10):e048101325

ISSN 2525-3409 | DOI: http://dx.doi.org/10.33448/rsd-v8i10.1325

o ISE tem adquirido uma maior percepção das questões ambientais, através das atividades e pesquisas que enfocam as temáticas do meio ambiente e a educação ambiental.

Palavras-chave: Sociedade. Meio Ambiente. Educação.

\begin{abstract}
The article, presented here, the result of a research, was to identify and describe the actions and ways of seeing environmental education (EA) in undergraduate courses of the Higher Institute of Education (ISE), an instance linked to the University Anápolis (UniEvangélica). Because it was a case study, the questionnaire was used as an instrument to collect data with teachers and students of undergraduate courses in chemistry, mathematics, biology, letters, history and pedagogy of the institution. The result was descriptive and exploratory, in which the qualitative discussion was discussed under the light of the environmental and environmental literature. As a result of the study, in the view of the subjects involved, the ISE has acquired a greater perception of environmental issues, through activities and research that focus on environmental issues and environmental education.
\end{abstract}

Key words: Society. Environment. Education.

\title{
1. Introducción
}

Teóricamente, en lo que se refiere a la concepción del mundo, se ha reforzado la idea de que el gran causante de los problemas ambientales se encuentra en el estilo de vida de los seres humanos y de la cultura hegemónica, que interfiere en el ambiente con el fin de proporcionar un sucesivo proceso de la construcción, apropiación y destrucción de los recursos naturales, proceso que fue impulsado por la cultura capitalista (Días, 2000; Leff, 2001; Mininni-Medina, 2001; Ramonet, 2003; Rocha, 2003; Sachs, 2002; Weil, 1990).

La cultura capitalista intervencionista, y poco comprometida con la armonía en la relación hombre/ambiente, intensificó la crisis ambiental. Esto generó, a finales de la década de 1960, muchas preocupaciones con el futuro del planeta. En este sentido, las preocupaciones, de cierta manera, forzaron a los eruditos a buscar formas para comprender los límites del actual modelo civilizatorio por medio de las intervenciones de los seres humanos en el ambiente (Dias, 2006; Ramonet, 2003).

Las inquietudes sobre el medio ambiente se intensificaron científicamente y esto demostró que las conferencias y los encuentros ambientales se volvieron cada vez más presentes en la sociedad y en la academia universitaria. Uno de los resultados discutidos, 
ISSN 2525-3409 | DOI: http://dx.doi.org/10.33448/rsd-v8i10.1325

procedentes de los estudios del medio ambiente, fue la reflexión de propuestas, medios y táctica que apuntan al desarrollo sostenible del planeta, con el objetivo de garantizar la supervivencia de las generaciones futuras (Barbosa, Bezerra, Abrahão y Pietrafesa 2007, Días, 2000, Días, 2006, Leff, 2001).

Estudiar la educación ambiental se vuelve pertinente mientras temática en el campo educativo debido a la urgencia de ampliación en las discusiones sobre de la inserción de las cuestiones del medio ambiente en el ámbito académico, en particular en los cursos de formación de profesores, así como la necesidad de ampliar las discusiones respeto de la EA en el ámbito de la enseñanza mayor.

Además, se subraya que este artículo es fruto de la mina disertación de maestría en Sociedad, Tecnología y Medio Ambiente de la UniEvangélica con la aprobación del Comité de ética e investigación bajo los siguientes registros: número de protocolo 04/2007 y oficio expedido por dicho órgano el número 034/2007 - CEP.

Siendo así esta investigación tiene como dimensión informar, a lo panorámico, aspectos del recorrido histórico de la educación ambiental (EA) en la enseñanza formal como elemento primordial para sensibilizar a las personas acerca de la importancia de la sostenibilidad y las temáticas del medio ambiente en la visión pedagógica. Para ello, se reflejos en este estudio, prioritariamente, las cuestiones ambientales y EA en los cursos de licenciatura del Instituto Superior de Educación del Centro Universitario de Anápolis, ya que son graduaciones en que las habilitaciones se dirigen al magisterio de la educación básica.

En el ámbito educativo se ha conferido empíricamente la relevancia de los docentes como sujetos fundamentales en la praxis docente, ya que la EA puede ser entendida como un conocimiento que colabora en la (re) construcción de una realidad ambiental sana y sostenible.

En esta misma línea de razonamiento, Jacobi (2005) confirma la necesidad de preparar a los docentes para la (re) elaboración de propuestas para una EA participativa en la que la transmisión, codificación, decodificación y externalización ocurra por medio de la mediación de los saberes y de la expresión significativamente sostenible. Ante el presente dilema coyuntural en torno a la dimensión ambiental y de la EA se asienta, en la postura de las personas y en la visión del mundo holístico, la no concepción del todo como la suma de las partes disociadas, sino un todo interconectado.

Al alejarse de la visión paradigmática moderna, fundamentada en la visión cartesiana, que valora la propuesta holística que se encuentra evidenciada en la misión institucional de la UniEvangélica, es que surge la preocupación con el desarrollo sostenible de la región. En este 
ISSN 2525-3409 | DOI: http://dx.doi.org/10.33448/rsd-v8i10.1325

sentido, este estudio verifica la importancia de la institución como colaboradora en la formación de los profesionales de la educación (licenciados) y de los ciudadanos conscientes con postura crítica y reflexiva en relación al medio ambiente.

En este sentido, el artículo, ahora presentado es un estudio de caso de cuño cualitativo, con algunos levantamientos cuantitativos. Primero se presenta, en el recorrido metodológico, aspectos relacionados a los delineamientos de la investigación, el escenario del estudio, los resultados y las discusiones.

Sin embargo, el objetivo de la investigación es identificar algunos aspectos existentes sobre la Educación Ambiental en la visión de los alumnos y de los profesores de las licenciaturas ofrecidas por la institución, además de informar el panorama histórico de la Educación Ambiental.

\section{Metodología}

El estudio se realizó en el Instituto Superior de Educación de la UniEvangélica, que se ubica en el municipio de Anápolis en el unidad federativa de Goiás. Es una ciudad de porte medio que está inserta en el eje comercial del Centro Oeste de Brasil. Situada entre las dos capitales, Brasília, capital Federal y Goiânia, la capital Estatal.

Figura 1: Localización de Anápolis, Goiânia y Brasília

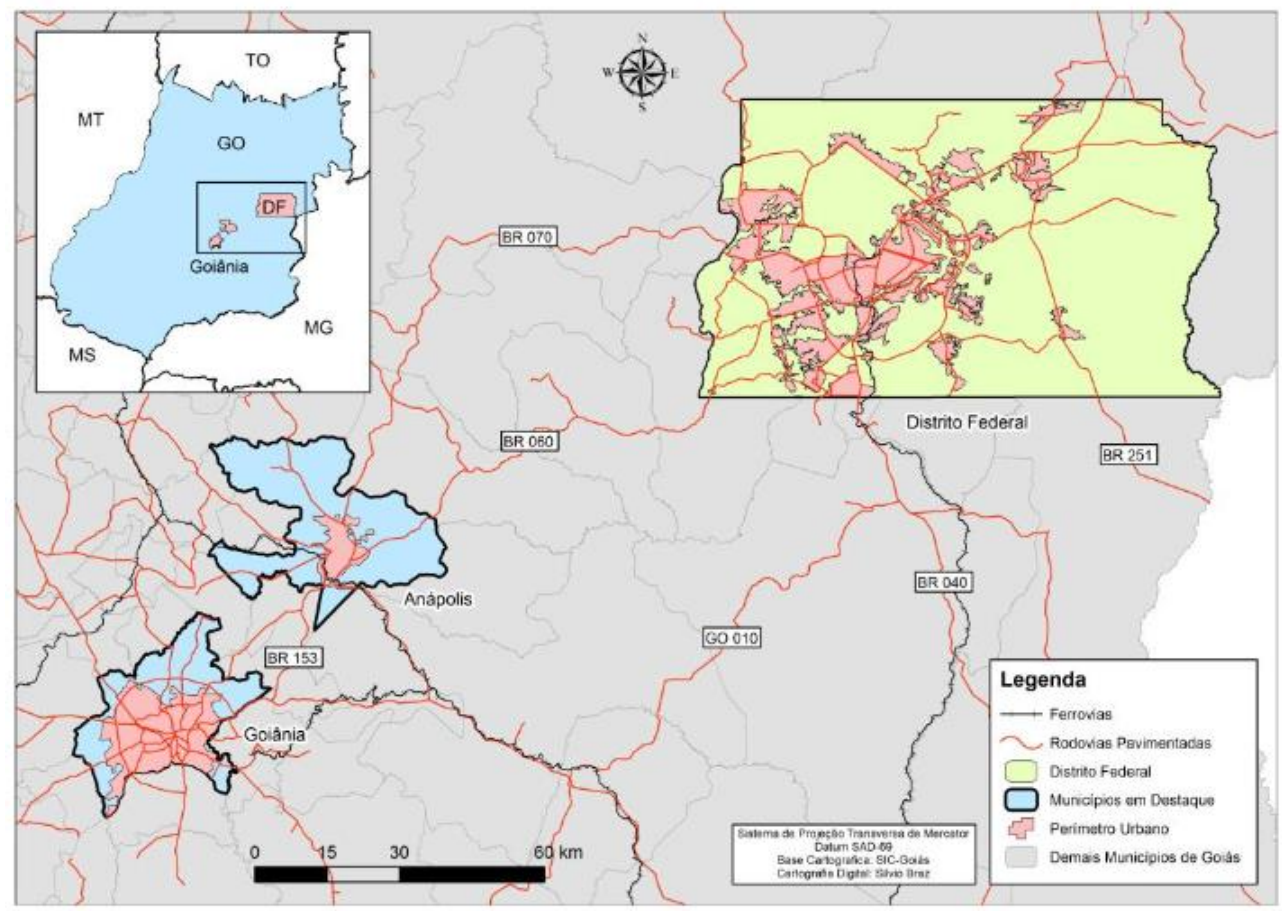

Fuente: Borba (2011 en Borba, Milagros y Barrera, s/d, p. 12) 
Los estudios de Castro (2004) y Polonial (2000, 2007) corroboran al señalar que el municipio de Anápolis, en su trayectoria sociohistórica, posee una identidad envuelta en la vocación comercial. Considerada la capital industrial del Centro Oeste de Brasil, y del Cerrado, se encuentra con una ubicación estratégica entre Brasilia y Goiânia, capital federal y capital estatal, respectivamente. Esto indica la posibilidad de Anápolis de mediar las negociaciones y facilitar el crecimiento de las diversas regiones de Brasil.

Es justamente en esta región socio-geográfica que se encuentra instalada la Asociación Educativa Evangélica (AEE), mantenedor de instituciones de educación a nivel básico y superior (UniEvangélica). Fundada en 1947, por medio de la instalación del Colegio Couto Magalhães, que en la época tenía el propósito de ser un campo complejo educativo, con una filosófica cristiana-evangélica, tenía el objetivo de ofrecer enseñanza de calidad para los sujetos de la región

Consolidado en la educación básica y siendo referencia para el Estado de Goiás por la oferta de una educación de calidad, se creó la primera facultad de Anápolis, la Facultad de Filosofía Bernardo Sayão. Por tratarse de una institución de enseñanza superior pionera en la ciudad, la actuación se inició en la década de 1960 con la creación de los primeros cursos que apunta a la formación de profesores, y con el paso de los años se ha adaptado para atender a las demandas sociales de la sociedad hoy en día.

En la última década del siglo pasado, la AEE se desarrolló, ampliándose, y lo que antes eran facultades aisladas - Facultad de Filosofía, Facultad de Odontología y Facultad de Derecho -, se unieron por fuerza regimental unificado, pasó a tener la nomenclatura "Facultades de la Asociación Educativa Evangélica" (FAEE). En este raciocinio, además de los cursos de licenciatura, derecho y odontología, se crearon nuevos cursos para prestar servicios a la comunidad, con valores cristianos y en la promoción de la ciudadanía y de su inserción en la sociedad (UniEvangélica, 2006).

Actualmente la institución es Centro Universitario, sus instalaciones se expandieron, y se crearon nuevos cursos de graduación y postgrado para ofrecer una mayor demanda en la educación del municipio y en la región circundante. (UniEvangélica, 2006).

En este contexto, la investigación se trata de un estudio de caso, que tuvo el objetivo de identificar y describir los aspectos que se relacionaron con la EA, en los cursos de licenciatura del ISE de la UniEvangélica, ya que son cursos de graduación que habilitan a los estudiantes profesionales para el magisterio de la educación básica. 
ISSN 2525-3409 | DOI: http://dx.doi.org/10.33448/rsd-v8i10.1325

La población investigada, por medio de aplicación de cuestionarios, involucró a los profesores y alumnos de las Licenciaturas en: Biología, Historia, Letras, Matemáticas, Pedagogía y Química. Para participar en la investigación, fueron incluidos los profesores y alumnos que se dispusieron a participar voluntariamente de la investigación, respondiendo las preguntas del cuestionario. Se excluyeron a los sujetos que no entregaron los cuestionarios contestados en el período de seis meses.

La Tabla 1 presenta los datos cuantificadores conexo a la población y el muestreo de los sujetos que participaron voluntariamente en la investigación.

Tabla 1 - Muestreo de sujetos involucrados en la investigación

\begin{tabular}{|l|cccc|cccc|}
\hline $\begin{array}{l}\text { Cursos de } \\
\text { Licenciatura }\end{array}$ & $\begin{array}{c}\text { frecuencia } \\
\text { población }\end{array}$ & $\%$ & $\begin{array}{l}\text { frecuencia } \\
\text { muestreo }\end{array}$ & $\%$ & $\begin{array}{c}\text { Maestros } \\
\text { frecuencia } \\
\text { población }\end{array}$ & $\%$ & $\begin{array}{c}\text { frecuencia } \\
\text { muestreo }\end{array}$ & $\%$ \\
\hline Biología & 107 & 100 & 61 & 56 & 16 & 100 & 6 & 37,5 \\
\hline Historia & 59 & 100 & 28 & 47,8 & 11 & 100 & 4 & 36,3 \\
\hline Letras & 181 & 100 & 58 & 32 & 14 & 100 & 5 & 35,7 \\
\hline Matemáticas & 108 & 100 & 39 & 36,1 & 11 & 100 & 5 & 45,4 \\
\hline Pedagogía & 173 & 100 & 52 & 30 & 14 & 100 & 6 & 42,8 \\
\hline Química & 43 & 100 & 28 & 41,8 & 7 & 100 & 3 & 42,8
\end{tabular}

Fuente: Búsqueda de campo

Después de la recepción de los cuestionarios contestados por los sujetos investigados, se elaboró el proceso de verificación y análisis de los datos en tres momentos. En el primer momento, fueron tabuladas las cuestiones objetivas, en que a través del uso de la estadística descriptiva, se cuantificaron las respuestas obtenidas para mejor discutir las informaciones. En el segundo momento, el análisis de los datos se dio de forma cualitativa, desarrollada en cuatro pasos.

En el primer paso, se realizaron lecturas de las respuestas obtenidas de los alumnos y profesores, y fueron recortadas las palabras principales, que traducían la esencia de las respuestas de las preguntas, mientras que en el segundo paso se agruparon los recortes por afinidades en las respuestas, por medio de la interpretación literal de las informaciones obtenidas en los cuestionarios.

En el tercer paso, se organizó un cuadro de categorías, para mejor exponer las respuestas de los alumnos y profesores, y en el cuarto y último paso se realizó el análisis de las respuestas, bajo las luces de los autores que se encuentran referenciados en el texto. 
Res., Soc. Dev. 2019; 8(10):e048101325

ISSN 2525-3409 | DOI: http://dx.doi.org/10.33448/rsd-v8i10.1325

El cuarto, y último paso, ocurrió después del análisis de los cuestionarios, en el que se expusieron algunas observaciones acerca de las prácticas institucionales, dirigidas a las responsabilidades sociales y ambientales.

\section{Análisis y discusión de los resultados}

Los estudios de la literatura científica reforzaron la idea del modo hegemónico y descomprometido de los seres humanos provenientes de una cultura dominadora, destructiva y apropiada de los recursos naturales. Con las constantes denuncias y con la consolidación de las ciencias ambientales, ha poco a poco consolidado y sensibilizado nuevos estudiosos a investigar el medio ambiente local y regional en el intento de comprender los límites del modelo económico y civilizatorio.

Así, las preocupaciones ambientales se intensificaron en los medios científicos, sociales, políticos, económicos, académicos, etc. Por lo tanto, uno de los resultados de los estudios teóricos apuntó a la adquisición de una nueva forma, que subvertiría la economía dominante, y pasaría a vislumbrar estrategias que colocarían las acciones humanas en el contexto del desarrollo sostenible.

Sin embargo, en cuanto a los análisis de las respuestas de las cuestiones objetivas de los cuestionarios, que al preguntar a los estudiantes si el ISE o UniEvangélica desarrolla, o desarrolló, directa o indirectamente, la interacción de los saberes ambientales, pedagógicos y científicos, el resultado cuantitativo constata que los cursos de la biología (83,6\%), Historia $(82,1 \%)$, Letras (79,3\%), Matemáticas (60\%), Pedagogía (53,9\%) y Química $(77,8 \%)$ evidencian que la institución desarrolla la interacción de los saberes, y que esta actividad ocurre en la forma de conferencias, seminarios y actividades de extensión.

En este sentido, la existencia de propuestas que se relacionan con la interdisciplinariedad, la transversalidad y la multidisciplinariedad en la formación académica, fueron percibidas solamente en los cursos de Biología y de Pedagogía.

La interdisciplinariedad como táctica de enseñanza-aprendizaje para la educación ambiental puede ser orientada hacia una finalidad práctica, por trascender la simple unificación de los saberes expande nuevos caminos indispensables para el proceso de reorganización de nuevos saberes, métodos, técnicas y producción de conocimiento innovadores y transformadores (Leff, 2001).

En cuanto a la transversalidad Minnini-Medina (2001) afirmó que la integración de los saberes con el medio ambiente, como directriz pedagógica debe ser de una exterioridad del 
Res., Soc. Dev. 2019; 8(10):e048101325

ISSN 2525-3409 | DOI: http://dx.doi.org/10.33448/rsd-v8i10.1325

universo educativo y al mismo tiempo debe permear las disciplinas formales en la enseñanza. Es en este sentido que la transversalidad proporciona un entendimiento integral de los diversos objetos de los saberes y del conocimiento para la ampliación del sujeto epistémico. La producción del conocimiento transversal supera la dicotomía entre sujeto y objeto. Así, la transversalidad, por esa vía, proporciona un escenario para la inclusión de saberes que van más allá de las aulas de las escuelas y elabora sentidos, significados y significancias en el cotidiano de los alumnos.

Sin embargo, Zucchi (2002) afirmó que en sentido práctico la propuesta multidisciplinaria tiene el propósito de reunir resultados ya obtenidos por los saberes/conocimientos de la ciencia, mediante el enfoque disciplinario. Dicho de otro modo, los Expertos, profesores y profesionales se reúnen y colaboran, cada uno con sus saberes instrumentalizados/de la ciencia, para la resolución y/o minimización de un determinado problema. Así, la postura multidisciplinaria, que es la unión dialogada de estudios interconectados por diversos campos científicos, tiene el objetivo de unirse saberes y discutir entre sí para alcanzar una posible resolución de problemas y producción de nuevos saberes.

Sin embargo, en el recorrido de la investigación, al cuestionar a los profesores acerca de la existencia de prácticas pedagógicas que son más adecuadas para trabajar la EA y las cuestiones ambientales, se constata que el 44,4\% de los profesores del curso de biología afirmaron que la multidisciplinaridad es una buena alternativa para trabajar con las temáticas ambientales, mientras que en los cursos de historia (60\%), letras (40\%), matemáticas (80\%), pedagogía $(42,8 \%)$ y química $(50 \%)$, que los docentes creen que para trabajar con EA, de modo más relevante, es la propuesta de la interdisciplinaridad.

Zucchi (2002) aclaró que en lo tocante a la visión fragmentada la interdisciplinaridad se contradice con la visión unitaria, rígida, tradicional y disciplinada de las acciones y de las prácticas pedagógicas instrumentalizadas. Esta postura actúa como modos de transferir métodos de producción de saberes específicos de una disciplina a otra a fin de identificar nuevos modos de mirar y de reconocer objetividades de estudio. Así esa postura de enseñanza-aprendizaje es entendida como una forma de dialogar entre diversas formas de elaborar y construir conocimientos.

Sin embargo surgió la necesidad de la interdisciplinaridad como forma de praxis pedagógica integrativa de nuevos saberes. Según Minnini-Medina (2001) la interdisciplinaridad pone en jaque la visión cartesiana, fragmentada e instrumentalizada, en la cual el conocimiento humano de la modernidad se erigió. 
Res., Soc. Dev. 2019; 8(10):e048101325

ISSN 2525-3409 | DOI: http://dx.doi.org/10.33448/rsd-v8i10.1325

En la visión de los discursos, sólo el curso de Biología tuvo docentes que definieron, cosntextualizaron y trabajaron efectivamente con el término "medio ambiente". En los cursos de licenciatura en Química, Pedagogía, Historia, y nuevamente, Biología se trabajaron las cuestiones ambientales al menos una vez.

En este mismo nivel investigativo se constata que los estudiantes de Biología $(54,1 \%)$ y Química (77,8\%) indicaron que ya habían abordado profesores, en el aula, acerca de las cuestiones ambientales, mientras que los alumnos de Matemáticas (71\%), Pedagogía (59\%), Historia $(78,6 \%)$ y Letras $(69 \%)$ no hicieron siquiera este enfoque.

En cuanto a las actividades multidisciplinares e interdisciplinares, autores como Fazenda y Arantes et al. (1998), Leff (2001) y Minnini-Medina (2001) confirmaron que para que haya una actividad interdisciplinaria y/o multidisciplinar, es necesario que el profesor asuma la postura policompetente. La policompetencia exige que la práctica pedagógica establezca la conexión de los diversos saberes, guiados por la necesidad del diálogo de los diversos conocimientos, en que es indispensable para la reorganización de los saberes, métodos y técnicas de las diversas disciplinas que transformen sus conceptos.

Así, las propuestas de la EA, ya sea en la visión multidisciplinaria o interdisciplinaria, exigen una construcción y reconstrucción de conceptos y definiciones sobre el medio ambiente, los problemas ambientales y, consecuentemente, la reconstrucción de teorías y prácticas pedagógicas dirigidas a la EA.

En cuanto al desarrollo sostenible y la EA, cabe informar que el Cuadro 1 presenta los resultados de los recortes y de las agrupaciones realizadas por afinidades, sobre la base de la interpretación literal de las respuestas y de las informaciones obtenidas en los cuestionarios de los alumnos y de los profesores.

Cuadro 1 - Recortes y afinidades

$$
\begin{gathered}
\text { DATOS SIGNIFICATIVOS } \\
\text { Preguntas realizadas a los profesores y estudiantes } \\
1-\text { ¿Qué entiende por medio ambiente? } \\
2-\text { Qué entiende por problemas ambientales? } \\
3-\text { ¿Qué entiende por educación ambiental? } \\
\text { Respuestas obtenidas }
\end{gathered}
$$

1-Relación entre factores bióticos y abióticos; planeta; Recursos naturales; la naturaleza; la biodiversidad; la sociedad; Factores bióticos y abióticos; elementos que componen el medio en que vivimos y el lugar de relación de los seres.

2-Interferencia humana; Agresiones al Planeta; Causados por el hombre; Desequilibrio de la Naturaleza; Destrucción de la naturaleza; Cambios en el medio natural. Interferencia humana en el medio natural; Provocados por el hombre.

3-Conscientizar para preservar el medio ambiente; Conscientizar sobre las agresiones; Conscientizar sobre los problemas naturales; Preservar el medio ambiente; Conscientizar sobre la preservación del medio ambiente; Preservar el medio ambiente; Reflexionar sobre la temática en general; Conscientizar sobre los problemas con la naturaleza y Concienciar para preservar

Fuente: Investigación de campo 
De acuerdo con el cuadro 1, los sujetos tienen una visión simplista y genérica sobre el medio ambiente. Se entendió que están relacionados con los recursos naturales, la biodiversidad, el lugar en que los seres humanos se relacionan, y también es visto como factores bióticos y abióticos y su interrelación.

Ricklefs (2003) conceptualiza el medio ambiente como un sistema ecológico complejo, constituido por organismo, población, comunidad, ecosistema y por la biosfera del planeta. Y en ese complejo contexto se encuentra el ser humano.

En el cuadro 1 quedaron expuestas el entendimiento de los docentes y discentes sobre los problemas ambientales. En la visión de los sujetos investigados, son entendidos como aquellos que son oriundos por las acciones humanas, causando muchos riesgos a la salud humana. La destrucción de la naturaleza, la contaminación, el crecimiento desordenado de las ciudades, la contaminación de los recursos naturales, la erosión del suelo entre otras.

Así, la comprensión de los profesores y de los alumnos, están dirigidos por una visión antropocéntrica en la que los problemas ambientales provienen de las influencias y interferencias del hombre con el medio natural, evidenciando así el entendimiento de Weil (1990) sobre el origen de los problemas ambiental.

Ante las informaciones sobre los problemas ambientales, cabe decir de la urgencia y de la emergencia de la sostenibilidad insertada en todos los aspectos de la vida humana. De este modo, el discurso de la sustentabilidad, que aún se encuentra en proceso de construcción, se originó como presupuesto multirreflexivo y multidimensional, anhelando apuntar a los seres humanos el camino peligroso para el cual la sociedad se dirige.

En este sentido, el discurso de la sustentabilidad admite que los cambios, originarios de las actividades humanas, hicieron que el ambiente fuera modificado, dando lugar a los anhelos humanos. Otro período que se dice por las aspiraciones humanas de desarrollo económico y social, sin la preocupación por el medio ambiente. Esto coincide con la teoría de

Iannone (1992) al relatar que con la llegada de la Revolución Industrial en función de los múltiples impactos incidentes en la estructura de la sociedad. Hecho el que presionaba a los industriales y comerciantes a una mayor demanda y mayor calidad de ofertas de productos.

Por lo tanto, el pensamiento científico, económico y político no se preocupó mucho con los principios del medio ambiente y ni siquiera con la sostenibilidad ambiental. En el presente momento, los últimos años han sido fuertemente marcados por las cuestiones ambientales que han alcanzado reconocimiento en la sociedad. Es en ese contexto que surge la 
Res., Soc. Dev. 2019; 8(10):e048101325

ISSN 2525-3409 | DOI: http://dx.doi.org/10.33448/rsd-v8i10.1325

importancia en intentar alertar sobre ese camino. Así la EA tiene como herramienta estratégica, la promoción de la concientización humana para promover la concientización humana en la preparación de propuestas de políticas y de participación de la sociedad a fin de la valorización y la obtención de nuevos hábitos sostenibles.

En la visión de Días (2006), no existen dudas en cuanto a los nuevos mecanismos y modos de producción capitalista, en que se añade la explotación intensiva y sistémica de la explotación de la naturaleza potenciada por la revolución industrial. Esta cultura potencializada por la revolución se extendió de forma descontrolada y miope en cuanto a las consecuencias del desarrollo económico y degradante que proporcionó consecuencias para el medio ambiente. Los procesos de industrialización añadieron de forma espectacular, y fueron construidos con una racionalidad que no vislumbraba una visión holística del medio ambiente. Sin embargo, en Brasil las propuestas para insertar la EA en las instituciones de enseñanza superior surgieron en 1980 y una década después fue en Europa.

En lo que se refiere a la resolución de los problemas ambientales, se percibe que, en la visión de los sujetos informantes, la sociedad en general es la responsable por la búsqueda de la sostenibilidad. Así, la EA tiene gran importancia como componente de estudio que proporciona el despertar de la conciencia ambiental.

En esta dirección, Dias (2000) admite que la educación ambiental tiene una finalidad que va más allá de los saberes instrumentales que vienen de la biología. Ella tiene el objetivo de proporcionar y promover la comprensión de la existencia, en todos los sentidos, de la interdependencia social, ecológica, política y económica de la sociedad. También debe promover para todos los seres humanos la posibilidad de producir conocimientos, y sentir los valores, proporcionar subsidios para los cambios de hábitos y actitudes necesarios para proteger y mejorar la calidad del ambiente. Para ello, se hace necesario incorporar nuevos modos de conducta de los sujetos, de los grupos sociales y de toda la sociedad en su conjunto, a buscar alternativas y soluciones a los problemas derivados de la explotación desenfrenada y exploratoria del medio ambiente.

En cuanto a las informaciones sobre el entendimiento de la EA, los sujeitos de la investigación referenciaron que la EA trilla por tres aspectos fundamentales: concientización, reflexión y preservación.

En este sentido, Barbosa et al. (2007) informa que socialmente la educación ambiental debe insertarse, de modo formal y no formal, para que la esencia de la EA sea agente sensibilizadores, perceptibles, racionales, reflexivos, críticos y de actitudes políticas. Siendo 
así la EA emerge como una relevante posibilidad de discusión en la praxis como uno de los indicadores para el desarrollo sostenible.

Los discentes y docentes que participaron como sujetos informantes de la investigación, apuntaron que la UniEvangélica desarrollaron temáticas ambientales en el contexto académico a través de proyectos de investigación, extensión y actividades ambientales. Entre los más citados fueron: UniLimpa, UniRecicle y el UniVerde.

El "UniLimpa - Universidad Consciente" fue un proyecto desarrollado en la disciplina "Práctica profesional V" del curso de Biología. El objetivo fue de promover la interacción de los cuerpos discentes en la vivencia profesional. Los estudiantes tenían la atribución de concientizar a estudiantes, profesores y demás servidores de la institución acerca de la producción y destino de los residuos institucionales.

Los alumnos-agentes multiplicadores de las acciones ambientales fueron identificados por la vestimenta que identificaba la temática del proyecto, además de visitar aulas para divulgar la campaña sobre los cuidados que las personas debían tener con la basura, a fin de proporcionar la conservación y la preservación del medio ambiente.

Con el desarrollo de esta actividad, los discentes del curso de Biología se empeñaron para concientizar sobre la producción y destino de los residuos generados en la institución, la necesidad del reciclaje, del reaprovechamiento y los cuidados en preservar el medio ambiente.

El "UniVerde" fue un proyecto que proporcionó el rescate a un espacio de reserva natural para la realización de las actividades ambientales y la EA. Las actividades que fueron ofrecidas: taller de reciclaje de papeles, taller basura vira arte (con el reaprovechamiento de residuos sólidos), charlas educativas y también la Pista ecológica del tucán. El público objetivo fueron los alumnos de la educación básica y superior de Anápolis, y hubo la participación de otras entidades que se interesaron en visitar la senda ecológica.

El "UniRecicle" sobrepasó los espacios físicos de la UniEVangélica y se objetivó en la constitución de acciones ambientales a través de la educación y de la participación de la comunidad de barrios carentes de la ciudad de Anápolis, con el objetivo de proporcionar la sensibilización social sobre la importancia de la separación, reutilización reciclado y reutilización de los residuos sólidos. Basado en la promoción de la salud de los sujetos y la protección del medio ambiente-inspirada en los principios holíticos y en el programa de gestión de residuos sólidos, el proyecto contó con una red de líderes, socios, residentes, voluntarios y otras instituciones de la sociedad para proporcionar subsidios para las acciones educativas de reaprovechamiento y reciclaje. 
Res., Soc. Dev. 2019; 8(10):e048101325

ISSN 2525-3409 | DOI: http://dx.doi.org/10.33448/rsd-v8i10.1325

Con ello, se percibió que con los proyectos, tanto el UniLimpa como el UniVerde señalaron la postura de los alumnos y de los profesores de la licenciatura en Biología, y sirvieron como maneras sensibilizadoras y conscientizadoras a través de las actividades ambiental-sostenibles promovidas. Esto demostró que, en cierto modo, las actividades desarrolladas integró la integración de responsabilidades sociales y ambientales de la IES, pues tales actividades no se alcanzan sólo al público interno, por sobrepasar los muros de la Institución, como fue el caso del UniRecicle.

\section{Consideraciones finales}

El resultado del estudio ha corroborado que el ISE tiene, poco a poco, una percepción más volcada a las cuestiones ambientales, a través de actividades e investigaciones que enfocan las temáticas del medio ambiente. Así como incluso señaló la existencia de un estado elemental de las IES en la concepción de la toma de conciencia de los sujetos sobre su papel de responsabilidad ambiental. Concomitantemente, se verificó que la UniEvangélica ha insertado y legitimado a las acciones y prácticas mirando que, en el futuro, se construyan elementos imprescindibles en la posible formulación de directrices ambientales específicas de las IES.

Ante estas constataciones, se percibió que en relación a las prácticas de los profesores, aún no hay un trabajo efectivo que merece ser coordinado de forma diferenciada, multidisciplinaria e interdisciplinaria que agreguen las diferentes áreas de conocimiento. Las posturas multidisciplinares e interdisciplinarias son relevantes, como tácticas didácticas, por proporcionar un ambiente integrativo e interactivo de métodos y saberes / conocimientos diversificados, apuntando el acto de concientizar y de proporcionar hábitos, acciones, actitudes y costumbres sostenibles en la esfera ambiental.

Luego, en esta coyuntura, el acto de concientizar es visto como responsabilidad de todos, en todas las instancias sociales y humanas. Por lo tanto, el papel de la institución de enseñanza superior no es sólo el de transmitir informaciones técnicas y científicas, la responsabilidad está comprendida en la formación del ciudadano. Así, se sugiere que las prácticas de EA no se límite sólo a la sensibilización y la concientización. Es necesario agrandar por medio de las acciones y de las prácticas peculiares de cada curso, en formar profesionales comprometidos con los aspectos profesionales, humanos, sociales y ambientales. 


\section{Referências}

Barbosa, Ivaldete; Bezerra, Leonardo M., Abrahão, Viviane \& Pietrafesa, José Paulo (2007). Educação ambiental: uma análise exploratória. Educação \& Mudança, 18(19), 35-50.

Bezerra, Leonardo M. (2019). As avenidas da minha cidade: considerações a respeito das nomenclaturas. Research, Society and Development, 8(8):e27881215 disponible: https://rsd.unifei.edu.br/index.php/rsd/article/view/1215/989 . Acceso al: 01 de junio de 2019.

Borba, Odiones de Fátima, Milagres, Géssica Filgueiras \& Barreira, Celene Cunha Monteiro Antunes. (s/d). Anápolis/GO e suas interfaces com a região urbana do eixo Goiânia/GOBrasília/DF. In: Observatório Geográfico América Latina. s/d, disponible: http://observatoriogeograficoamericalatina.org.mx/egal14/Geografiasocioeconomica/Geografi aurbana/060.pdf. Acceso al: 01 de junio de 2019.

Castro, Joana D’Arc Bardella (2004). Anápolis: Desenvolvimento industrial e meio ambiente. Anápolis: AEE.

Dias, Genebaldo Freire (2000). Educação ambiental: Princípios e práticas. São Paulo: Gaia.

Dias, Reinaldo (2006). Gestão ambiental: Responsabilidade social e sustentabilidade. São Paulo: Atlas.

Fazenda, Ivani; Arantes, Catarina (1998). Didática e interdisciplinaridade. Campinas, SP: Papirus.

Fouto, Ana Rita Ferreira (2002). O papel das universidades rumo ao desenvolvimento sustentável: das relações internacionais as práticas locais. Campus Verde. Acceso al: Noviembre, 2006, Disponible: http://campus.fct.unl.pt/campusverde/W_RIA_ARFF.doc.

Iannone, Roberto Antônio (1992). A revolução industrial. São Paulo: Moderna.

Jacobi, Pedro Roberto (2005). Educação ambiental: o desafio da construção de um pensamento crítico, complexo e reflexivo. Educação e Pesquisa, 31(2), 233-250.

Kraemer, Maria Elisabeth Pereira. A universidade do século XXI rumo ao Desenvolvimento Sustentável. Ambiente Brasil. Acceso al: Noviembre, 2006. Disponible http://www.ambientebrasil.com.br/composer.php3?base=./educacao/index.php3\&conteudo=/e ducacao/artigos/universidade.html

Leff, Enrique (2001). Saber ambiental: Sustentabilidade, racionalidade, complexidade, poder. Petrópolis R.J.: Vozes.

Mininni-Medina, Nana (2001). Educação Ambiental na educação formal. In: Leite, Ana Lúcia Tostes de Aquino \& Mininni-Medina, Nana. Nana (orgs). Educação Ambiental: Curso básico à distância (pp 37-92). Brasília: DF

Polonial, Juscelino Martins (2000). Ensaios sobre a história de Anápolis. Anápolis: AEE. 
ISSN 2525-3409 | DOI: http://dx.doi.org/10.33448/rsd-v8i10.1325

Polonial, Juscelino Martins (2007). Anápolis: Das origens do povoado à revolução de 1930. In Toschi, Mirza Seabra (org.). 100 anos: Anápolis em pesquisa (pp. 15-34). Goiânia: Vieira.

Ramonet, Ignacio (2003). Guerras do século XXI. Petropolis: Vozes.

Rocha, Paulo Ernesto Diaz (2003). Trajetórias e perspectivas da interdisciplinaridade ambiental na pós-graduação brasileira. Ambiente \& Sociedade, 6(2), 155-18.

Sachs, Ignacy (2002). Caminhos para o desenvolvimento sustentável. Rio de Janeiro: Garamond.

Tauchen, Joel Antonio (2007). Um modelo de gestão ambiental para implantação em instituições de ensino superior. Dissertação de Mestrado em Engenharia de Produção, Universidade de Passo Fundo, Passo Fundo, Brasil.

Unievangélica (2006). Plano de Desenvolvimento Institucional: segundo aditamento. Anápolis, Goiás: AEE.

Weil, Pierre (1990). A arte de viver em paz: Por uma consciência e educação. São Paulo: Gente.

Zucchi, Odir Jose (2002). Educação ambiental e os parâmetros curriculares nacionais: Um estudo de caso das concepções e práticas dos professores do Ensino Fundamental e Médio em Toledo-Paraná. Dissertação de Mestrado em Engenharia de Produção. Universidade Federal de Santa Catarina, Florianópolis, Brasil.

\section{Porcentagem de contribuição de cada autor no manuscrito}

Leonardo Mendes Bezerra - 100\% 